thought to be susceptible to foreign influences.

And why did Vavilov not fight Lysenko earlier and more aggressively? Pringle demonstrates that Vavilov was guided by his student pledge to help the Soviet people and that he was committed to exploring all leads, however improbable, that might increase food production. Vavilov encouraged many scientists, including Lysenko, to test different approaches. Naively, Vavilov did not expect that Lysenko would play by political rather than scientific rules. At a 1948 session of the Lenin Academy, Stalin was so determined that Lysenko should triumph that he drafted Lysenko's opening remarks himself, emphasizing the correctness of Lamarckian thinking. A letter included in the official report ended: "Glory to the great Stalin ... coryphaeus of progressive science!"

Neither Vavilov nor his work featured in this session. Following the 1939 conference, Lysenko had progressively dismantled Vavilov's institute, but Vavilov had remained free even as criticism of him became ever more vituperative. Then, on 6 August 1940, while collecting plants in the Ukraine, Vavilov was seized by the Soviet secret police and taken to Moscow. Pringle's account of Vavilov's 11-month interrogation is horrifying. In July 1941, Vavilov and two colleagues were tried and sentenced to death. Vavilov's appeal to the Presidium of the Supreme Soviet was turned down, but a personal plea to the head of the secret police led to his sentence being commuted to life imprisonment. His colleagues were shot. Vavilov died of starvation on 26 January 1943 in a prison in Saratov, the city where he had begun his illustrious career 26 years before.

Even now, politics continues to trump good science, as is evident from the delays in reducing global carbon emissions. Pringle's very readable account is a timely reminder that public policies must be based on rational decisions drawn from the best data available.

Jan Witkowski is executive director of the Banbury Center and professor in the Watson School of Biological Sciences at Cold Spring Harbor Laboratory, New York 11724, USA. He is co-author of Recombinant DNA: Genes and Genomes. e-mail:witkowsk@cshl.edu

\title{
Betting on black holes
}

\author{
The Black Hole War: My Battle with Stephen \\ Hawking to Make the World Safe for \\ Quantum Mechanics \\ by Leonard Susskind \\ Little Brown: 2008. 416 pp. \$27.99
}

The idea of a black hole in space produced by the collapse of a massive star dates back to the 1930 s, but it was only in the 1960s that astrophysicists began to understand the details. Evidence now suggests that black holes do exist, and are a key element of the great cosmic story. Yet some of their predicted properties remain puzzling and threaten cherished physical laws.

One long-running conflict concerns the fate of the material that implodes to form a black hole. In his new book The Black Hole War, theoretical physicist Leonard Susskind of Stanford University, California, describes how he sparred with Stephen Hawking of the University of

Cambridge, UK, about this thorny issue.

Black holes earn their name because their gravity is so strong they trap even light, appearing black from the outside. According to Einstein's general theory of relativity, the ball of matter that implodes to create the hole continues to shrink, meeting an uncertain fate at the centre and leaving behind it a region of empty space. Because physicists believe nothing can go faster than light, no information or material should escape the hole. Practically all the information about the collapsed star would be lost from the outside Universe, making it impossible to tell whether the star was made of matter, antimatter or green cheese; once inside the hole, its external physical properties would be the same.

A twist arose in 1975 when Hawking argued that black holes are not perfectly black. By applying quantum mechanics to the formation process of a black hole, he predicted that it must radiate heat. Because heat is a form of energy, this radiance would have to be paid for by gradually reducing the hole's mass, because energy and mass are equivalent in general relativity. In time, the hole would evaporate away completely, leaving behind just heat energy, mostly in the form of photons, with a few other particles such as neutrinos and electrons.

On an immense timescale, vastly longer than the current age of the Universe, the black hole would convert the entire star into heat. Hawking concluded that the heat energy would look the same whatever the star was made of originally. Many physicists did not like this conclusion. A principle of quantum mechanics is that information is never created or destroyed in a quantum process. For example, if you throw an encyclopaedia into a furnace, it might seem that the information is irretrievably lost, turned into incoherent heat. According to quantum mechanics, however, the information is lost only for practical purposes. The infrared photons emitted by the embers still contain the original information, but in a hopelessly scrambled form that renders it inaccessible to us. Leading particle physicists, led mainly by Susskind and Gerard 't Hooft of the University of Utrecht in the Netherlands, declared that the same would be true of black holes - the information about the original star would be enfolded in the emitted heat. It might be jumbled up, but it should all be there.

Hawking begged to differ. I recall him going through the argument during a conversation in

\section{Endless Universe: Beyond the Big Bang - Rewriting Cosmic History}

by Paul J. Steinhardt and Neil Turok (Broadway, \$14.95, £8.99)

Two theoretical physicists challenge the widely accepted Big Bang Theory. Turok and Steinhardt explain how new developments in astronomy, cosmology and physics have led them to develop their own 'Cyclical Universe' theory to explain cosmic origins.

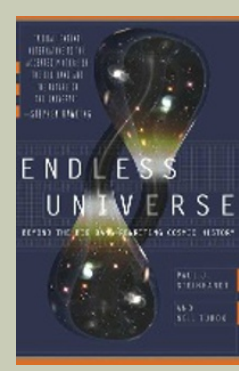

The Beginner's Guide to Winning The Nobel Prize: A Life in Science

by Peter Doherty (Columbia Univ. Press, E10.95)

Doherty's highly readable book describes how

to succeed in science and hopefully win that coveted prize. "For the aspiring young scientist, or a student considering a scientific career, Doherty opens the vault to the world of science, explaining how it works and how to get on," according to Peter Parham's review (Nature 443, 755-756; 2006).

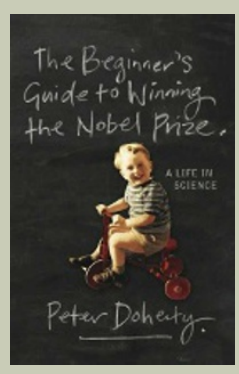


1978. He reasoned that crucial bits of information about subatomic particles cannot be carried by photons, so other types of particles such as electrons or quarks would also be needed. But there are not enough of these other particles in the radiation to embody all the information, because most of the heat energy from a black hole is in the form of photons. The conundrum became known as the information paradox. It may seem like theoretical nit-picking, but the paradox goes to the very heart of physical theory and its description of reality.

The momentous conclusion that a black hole swallows and permanently obliterates physical information didn't bother Hawking, whose background was in gravitational theory and space-time geometry rather than particle and quantum physics. With theorist Roger Penrose of the University of Oxford, UK, he proved that space and time could have boundaries or edges, called singularities, at which information might enter or leave the Universe. The general theory of relativity predicts that such a singularity lurks at the centre of a black hole, where the gravitational field and space-time warp become infinite. As a consequence, the imploding star's information might disappear from space and time through the hole's singularity. Hawking was sufficiently confident to place a bet with theoretical physicist John Preskill at the California Institute of Technology in Pasadena.

An uncertainty in Hawking's argument was the fate of the singularity. If the black hole evaporated completely then the singularity must presumably disappear too, but the details depend on an incomplete field of physics known as quantum gravity. In recent years, work in string theory - to which Susskind has made seminal contributions - has placed quantum gravity on a more secure foundation. Armed with such arguments, Susskind and others gathered support for the position that information is conserved, against Hawking's claim. In 2004, Hawking called a press conference and announced to the world that he had changed his mind. Black holes did not, after all, irreversibly annihilate information, he said. The bet with Preskill was duly settled in the form, appropriately enough, of an encyclopaedia.
The Black Hole War charts the ups and downs of this lengthy yet good-natured dispute. Susskind skilfully explains the subtleties of the physics that underlie the issue, and includes anecdotes to enliven the technical details. He has stuck to his guns for many years, but nevertheless resists the temptation to gloat over Hawking's eventual capitulation.

Is the matter laid to rest? I don't think so. Hawking justified his reversal by sketching out a calculation, but quantum gravity is still too unrefined for a rigorous proof. The weak point is that, in quantum gravity, the singularity can be replaced by a space-time region with a complicated and changing topology, allowing information to shift from one region of space- time into another disconnected one, perhaps from our Universe to a newly born 'baby universe'. Susskind dismisses this possibility, but the matter is far from resolved. It may be that if we consider the entire 'meta-verse' of all spatial regions, information is never lost. But if we restrict attention to a single universe, or connected region of space, then information can in fact leak out. Deciding the matter is a task for a future generation of theoretical physicists.

Paul Davies is director of the Beyond Center for Fundamental Concepts in Science at Arizona State University, Tempe, Arizona 85287-6505, USA. His latest book is The Goldilocks Enigma: Why Is the Universe Just Right for Life? e-mail:paul.davies@asu.edu

\section{Maths and mad hatters}

\section{Lewis Carroll in Numberland: His Fantastical Mathematical Logical Life by Robin Wilson \\ Allen Lane/Norton: 2008. 237 pp/208 pp. $€ 16.99 / \$ 24.95$}

Legend has it that Queen Victoria was so enchanted by Alice's Adventures in Wonderland that she insisted on Lewis Carroll's next work being sent to her. One can imagine her expression as she opened the book that arrived, entitled An Elementary Treatise on Determinants.

Charles Lutwidge Dodgson had many careers. He is best remembered for the sublime nonsense verse he wrote under the name Lewis Carroll. He was a pioneering childrens' photographer and a lay clergyman admired for his sermons. Before all else he was a mathematician who taught generations of students at the University of Oxford, UK, contributed to the fields of geometry, algebra and logic, and used games and puzzles to entertain and instruct. In Lewis Carroll in Numberland, mathematician Robin Wilson reveals Dodgson to be the grandfather of recreational mathematics.

He was precocious, orthodox and craved variety. Born in 1832 in Cheshire, UK, Dodgson was a lecturer at Oxford by his early twenties. At a time when non-Euclidean geometries were catching on, he wrote a four-act play stubbornly arguing that Euclid should remain at the centre of the Oxford curriculum. He invented a method to find the determinants of large matrices, but his strange notation meant that it never caught on. Later, he sought mathematical remedies for real injustices, suggesting tie-break methods for parliamentary elections to his friend Lord Salisbury, and devising a way to make lawn tennis tournaments fairer to the runners-up.

Some work was ahead of its time, especially his efforts to bring mathematics to young people. Although pupils complained of his "singularly dry and perfunctory manner" in the classroom, Dodgson's gift for teaching shone through in dozens of self-published guides for students, and in his letters to children. Wilson shows that he found humour in the plainest of subjects and did not underestimate his young correspondents, once commenting that intelligence seemed to vary inversely with size. In person, he drew their attention using guessing games and feats of memory. He could recite the first 71 digits of pi using a series of nonsense couplets as memory aids, and once contrived
Faust in Copenhagen: A Struggle for the Soul of Physics and the Birth of the Nuclear Age by Gino Segrè (Pimlico, E12.99)

Segrè relays the 1932 gathering of seven leading physicists that "symbolized the end of the political neutrality of physics and physicists, coinciding as it did with the arrival of Hitler and crucial discoveries in nuclear physics that would make possible the subsequent development of the atomic bomb", wrote Finn Aaserud (Nature 448, 869-870; 2007).

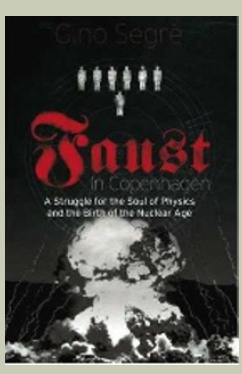

The Annotated Flatland: A Romance of Many Dimensions

by Edwin A. Abbott. Introduction and notes by lan Stewart (Perseus, \$17.95, £10.99)

Abbott's satirical tale of A. Square's journey to The Land of Three Dimensions is annotated by mathematician lan Stewart, providing helpful background on this maths fantasy. Abbott's ideas were thought radical by the Victorians but are now commonplace in both science and science fiction.

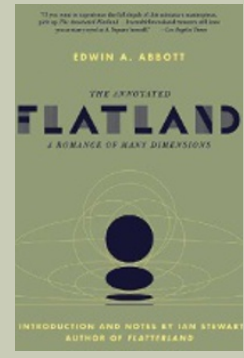

